\title{
Analysis of the ship target detection in high-resolution SAR images based on information theory and Harris corner detection
}

\author{
Yangyang Deng ${ }^{1}$, Haijiang Wang ${ }^{1 *} \mathbb{D}$, Shuo Liu', Min Sun ${ }^{1}$ and Xiaohong Li
}

\begin{abstract}
In order to make up the shortcomings of some existing ship target detection algorithms for high-resolution synthetic aperture radar (SAR) images, a ship target detection algorithm based on information theory and Harris corner detection for SAR images is proposed in this paper. Firstly, the SAR image is pretreated, and next, it is divided into superpixel patches by using the improved simple linear iterative clustering (SLIC) superpixel generation algorithm. Then, the self-information value of the superpixel patches is calculated, and the threshold $T_{1}$ is set to select the candidate superpixel patches. And then, the extended neighborhood weighted information entropy growth rate threshold $T_{2}$ is set to eliminate the false alarm candidate superpixel patches. Finally, the Harris corner detection algorithm is used to process the detection result and the number of the corner threshold $T_{3}$ is set to filter out the false alarm patches, and the final SAR image target detection result is obtained. The effectiveness and superiority of the proposed algorithm are verified by comparing the proposed method with the results of constant false alarm rate (CFAR) detection algorithm combined with morphological processing algorithm and other ship target detection algorithms.
\end{abstract}

Keywords: SAR image, Ship detection, CFAR, Superpixel, Information theory and Harris corner

\section{Introduction}

Synthetic aperture radar imaging is not limited by weather, illumination, or other conditions. To carry out research on ship SAR target detection is very important for marine monitoring management and timely military information acquisition [1].

There are many methods for ship target detection, but the constant false alarm rate (CFAR) detection algorithm is most widely used [2]. Scholars have proposed some detection methods based on different statistical distribution model of sea clutter in SAR images, including the gamma distribution model, Weibull distribution model, and so on [3-5]. However, they are not independent on the clutter statistical model, and they are not robust. Moreover, CFAR detection methods are based on pixel-level

\footnotetext{
*Correspondence: whj@cuit.edu.cn

${ }^{1}$ College of Electronic Engineering, Chengdu University of Information

Technology, Chengdu 610225, Sichuan, China

Full list of author information is available at the end of the article
}

detection, and they will cause some false alarm detection targets. After the concept of superpixel is proposed, the target detection in SAR images based on superpixel has developed rapidly [6]. It uses the superpixel instead of the pixel as the processing unit, and it can obtain a better detection result [7].

In recent years, the idea of visual attention has been introduced into SAR target detection and it has obtained some good performance. Hou et al. [8] proposed a ship detection method in SAR images based on visual attention model according to the prior knowledge that ships are in the water region rather than on the land, the ship detection performance is well, and the ship detection performance can be improved even though there are still some false alarm targets and some lost ship targets in the detection result. Liu and Cao [9] have combined the pyramid model of visual attention with singular value decomposition (VA-SVD) to detect the targets in SAR 
images; however, the computation speed of the algorithm is very slow, and the detection performance is not very good when the SAR images have a large and complex scene. Wang et al. [10] proposed a visual attention-based target detection method for high-resolution SAR images in complex scenes; even though the detection rate in complex is high, the original morphology of the targets cannot be well preserved in the final detection result.

As for the detection method based on information, Cao et al. [11] proposed a fast target detection method for high-resolution SAR images based on variance weighted information entropy (VWIE), and Wang and Chen [12] have used the method based on multiscale variance weighted image entropy (MVWIE) method to detect the ship targets in the complex background SAR images. They are proved to be effective to detect the true targets successfully; however, the discrimination of the target and the surrounding cells is difficult, and there are usually some false alarm targets in the detection result.

In order to improve the detection performance of the existing ship target detection method for highresolution SAR images, a ship target detection algorithm based on information theory and Harris corner detection for high-resolution SAR images is proposed in this paper.

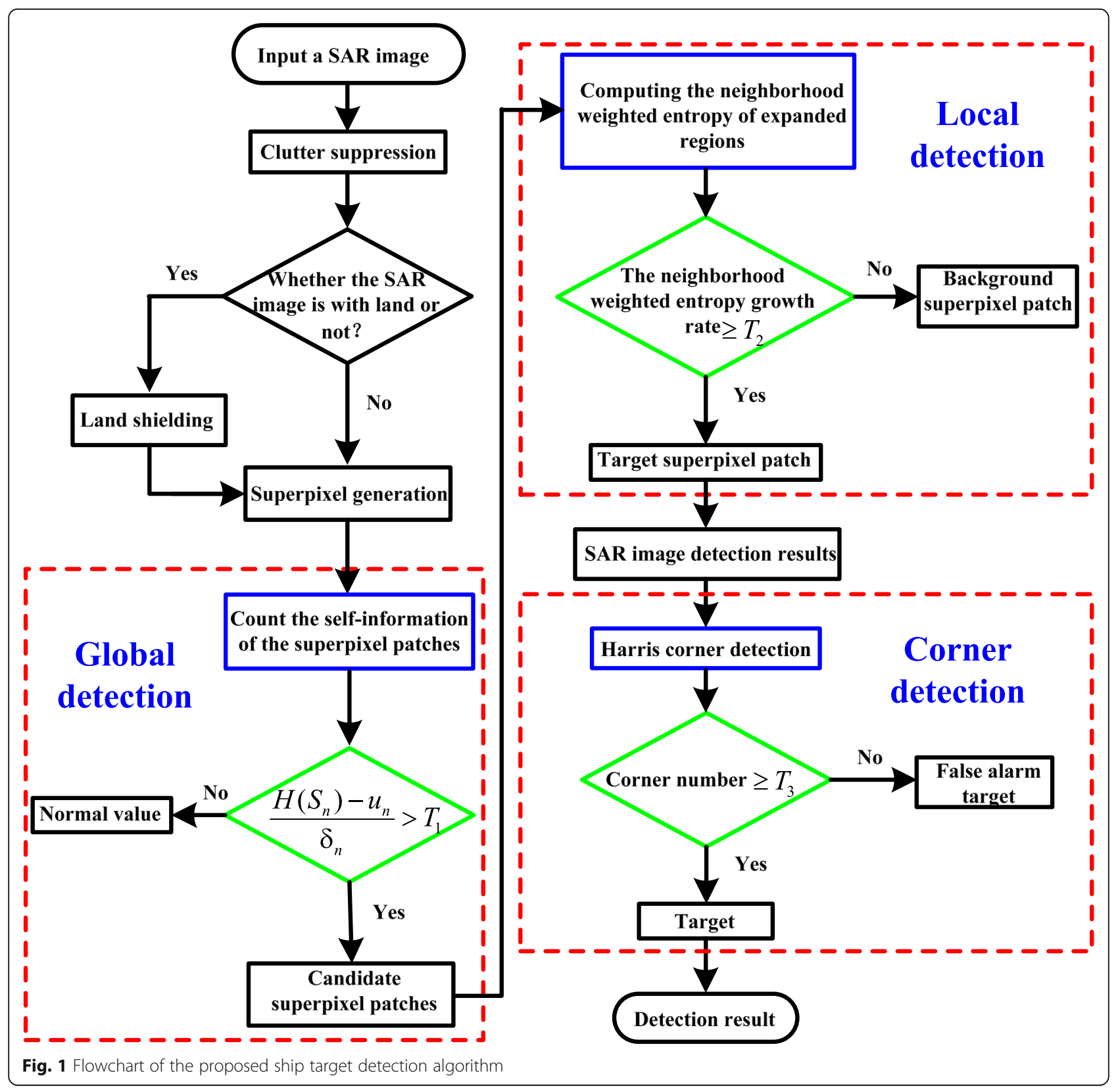




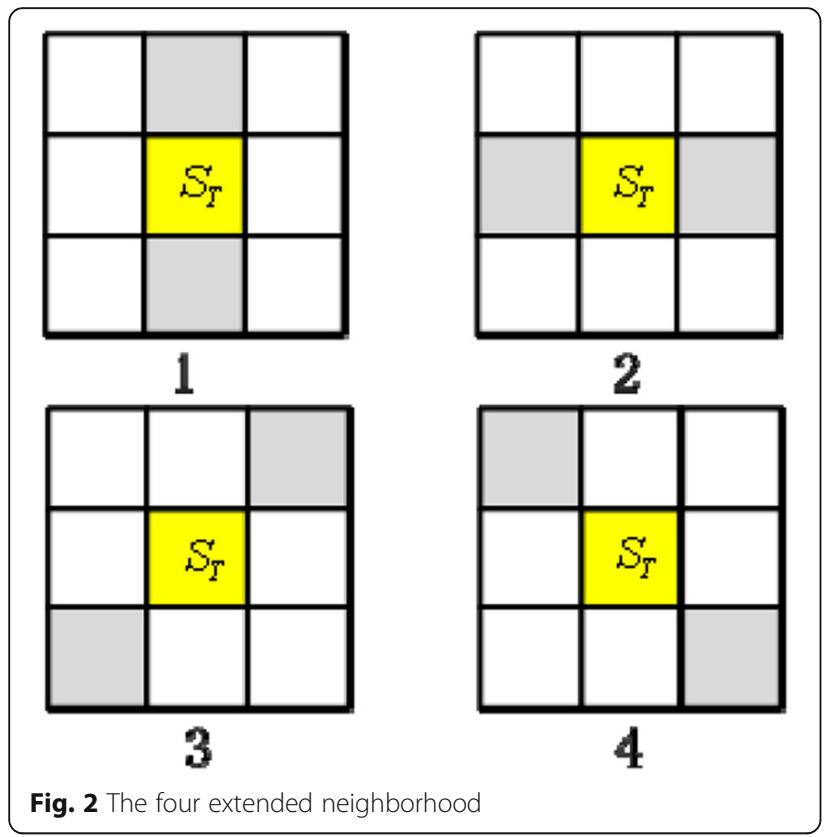

The remainder of this paper is structured as follows. Section 2 introduces the detection method based on information theory and Harris corner detection. Section 3 shows the details of our experiments and gives the result analysis based on the proposed method. Finally, the conclusion is given in Section 4 .

\section{Methods}

The proposed detection method consists of three stages, including the global detection, local detection, and Harris corner detection, as illustrated in Fig. 1.



Fig. 3 Original SAR image

\subsection{Superpixel generation algorithm}

At present, the superpixel generation algorithms such as watershed algorithm, mean shift algorithm, and $K$-means algorithm are widely used in the optical image processing. For ship target detection in SAR images, the subsequent processing will be easier if the edge of the superpixel patches can coincide or almost coincide with the edge of the targets in the SAR image. The simple linear iterative clustering (SLIC) superpixel generation algorithm [13] which is based on clustering can meet the requirement mentioned above. However, because the SLIC algorithm is used for optical image processing, without considering that the SAR image is the gray image, the color distance of an optical image must be adjusted to the grayscale. The other steps are the same as the SLIC algorithm.

\subsection{Self-information of SAR image}

In the information theory, the self-information of a random event $e_{i}$ is defined as:

$$
H\left(e_{i}\right)=-\log _{m} p\left(e_{i}\right)
$$

Among the expression, $p\left(e_{i}\right)$ is the occurrence probability of the random event $e_{i}$, and the variable $i$ represents the $i$ th possible event. If the base of the above logarithm is 2, the unit of $H\left(e_{i}\right)$ is bits.

For any superpixel patch $S_{n}$ in SAR image I, assuming that the superpixel patch is composed of $a \times a$ pixels, the intensity distribution model of $S_{n}$ can be expressed by the following formula:

$$
\left(\begin{array}{c}
r \\
p(r)
\end{array}\right)=\left(\begin{array}{llll}
0, & 1, & \ldots, & 255 \\
p(0), & p(1), & \ldots, & p(255)
\end{array}\right)
$$

Among the expression, the variable $p(r)$ represents the probability of the pixel whose grayscale intensity value is $r$. The likelihood function is used to express the probability of each superpixel patch. Its conditional probability value is:

$$
P\left(S_{n} \mid \mathrm{I}\right)=\prod_{k=1}^{a^{2}} P_{I}\left(S_{n}(k)\right)
$$

In the expression, $P_{I}(\bullet)$ is the probability distribution of the SAR image I, and $S_{n}(k)$ is the corresponding gray value of the $k$ th pixel. Because the order of pixels in superpixel patches is not considered, the upper expression can be represented by the probability distribution of superpixel patch $S_{n}$.

$$
P\left(S_{n} \mid \mathrm{I}\right)=\prod_{i=1}^{255}\left(P_{I}(i)^{P_{S n}(r) \cdot a^{2}}\right)
$$




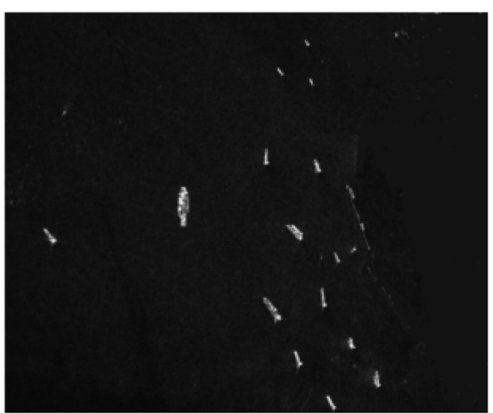

a

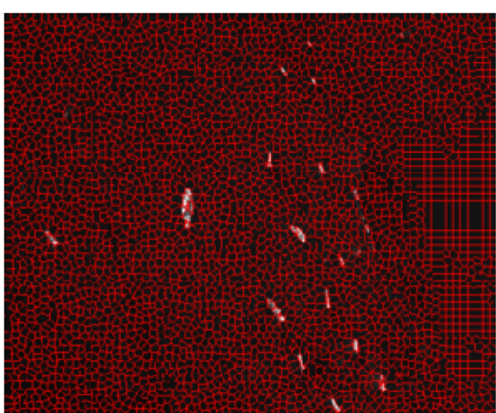

b

Fig. 4 The superpixel generation result of the original SAR image. a The land shielding result. b Superpixel segmentation result

Substituting Eq. (4) to (1), the corresponding self-information of the superpixel patch $S_{n}$ can be obtained, as illustrated in the following expression:

$$
\begin{array}{r}
H\left(S_{n} \mid \mathrm{I}\right)=-\log _{m}\left(P\left(S_{n} \mid \mathrm{I}\right)\right)=-\log _{m}\left(\prod_{r=0}^{255}\left(P_{I}(r)^{P_{S n}(r) \cdot a^{2}}\right)\right. \\
=-a^{2} \cdot \sum_{r=0}^{255}\left(P_{S n}(r) \cdot \log _{m}\left(P_{I}(r)\right)\right)
\end{array}
$$

A definition is made that if $P_{S n}(r) \neq 0, P_{S n}(r)=1$. Then, it can be obtained that:

$$
H\left(S_{n} \mid \mathrm{I}\right)=-a^{2} \cdot \sum_{r=0}^{255} \log _{m}\left(P_{I}(r)\right)
$$

In this way, the self-information of any superpixel patch $S_{n}$ can be calculated by using the probability distribution of the grayscale intensity value for the whole image.

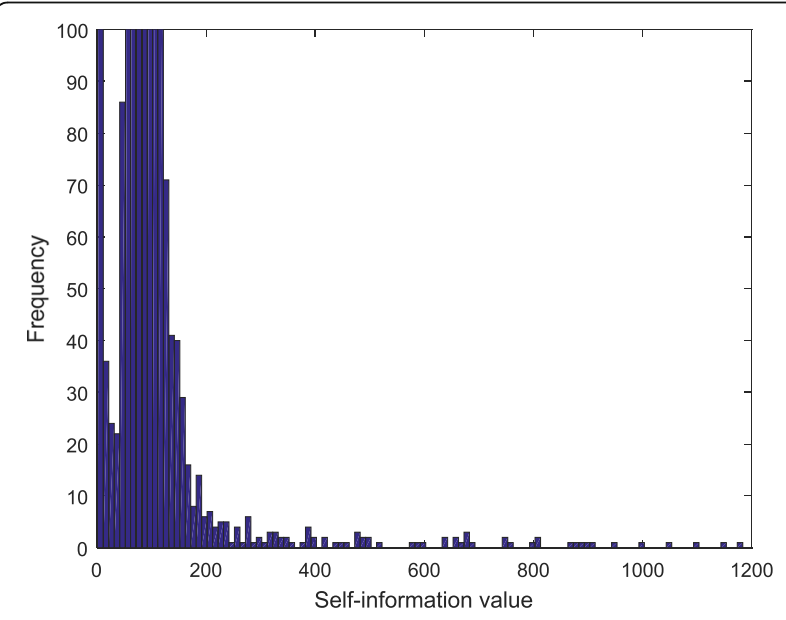

Fig. 5 The self-information statistics graph of the superpixel patches

\subsection{Outlier detection and false alarm filtering}

\subsubsection{Outlier detection}

Firstly, the self-information of all the superpixel patches is counted, and the corresponding distribution histogram is established. The outlier detection expression for the self-information of the superpixel patches is shown as follows:

$$
\left\{\begin{array}{l}
\frac{H\left(S_{n}\right)-u_{n}}{\delta_{n}}>T_{1}, \quad n \in(1,2, \cdots w), H\left(S_{n}\right) \text { is outlier } \\
\frac{H\left(S_{n}\right)-u_{n}}{\delta_{n}}<T_{1}, \quad n \in(1,2, \cdots w), H\left(S_{n}\right) \text { is normal }
\end{array}\right.
$$

In the expression, $H\left(S_{n}\right)$ is the self-information of the superpixel $S_{n}$ and assume that the number of superpixel patches is $w$. The variables $u_{n}$ and $\delta_{n}$ are respectively the mean and variance of the histogram distribution of the self-information corresponding to the $n$ superpixel patches, and the variable $T_{1}$ is the detection threshold. After the global detection, there may be some background superpixel patches in the candidate superpixel patches, and they can be filtered by using the following methods.

\subsubsection{The false alarm filtering method based on weighted information entropy}

By using $S_{T}$ as the center, the four extended $3 \times 3$ neighborhood $S_{T}(d)$ in the vertical direction, horizontal direction, right diagonal direction, and left diagonal direction can be obtained. The extended neighborhood areas are shown in Fig. 2.

The information entropy reflects the average amount of information in the image, and it can be expressed as:

$$
H=-\sum_{r=0}^{255} p(r) \log p(r)
$$




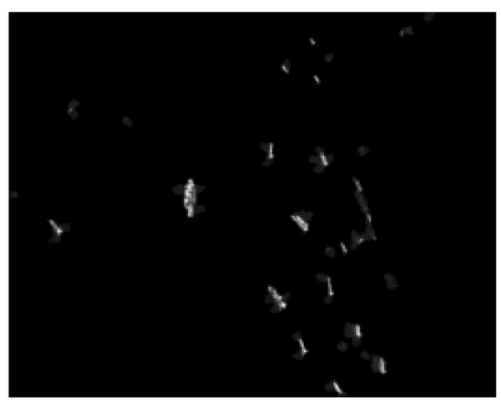

a

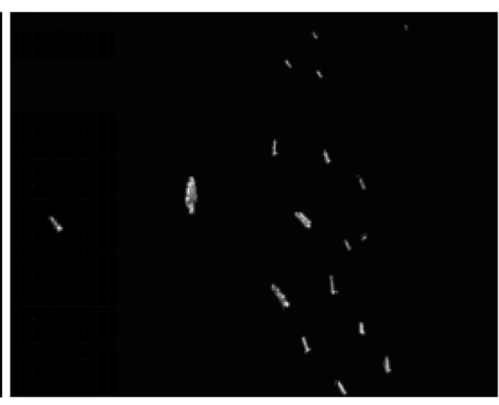

b

Fig. 6 The detection result based on information theory. a The candidate superpixel patches. b The weighted entropy filtering result

When $p(r)=0$, let $p(r) \log p(r)=0$. Among the expression, $p(r)$ represents the appearance probability of the pixels whose grayscale intensity value is $r$. The weighted information entropy of the testing superpixel patch $S_{T}$ can be expressed as follows:

$$
E\left(S_{T}\right)=-\sum_{r=0}^{255}\left(r-\overline{r_{V}}\right)^{2} P_{S_{T}}(r) \log _{2}\left(P_{S_{T}}(r)\right)
$$

The variable $\overline{r_{V}}$ is the mean grayscale intensity value, and the weighted information entropy $E\left(S_{T}(d)\right)$ of the extended neighborhood $S_{T}(d)$ in four directions can be expressed as follows:

$$
E\left(S_{T}(d)\right)=-\sum_{r=0}^{255}\left(r-\overline{r_{E R}(d)}\right)^{2} \cdot P_{S_{T}(d)}(r) \log _{2}\left(P_{S_{T}(d)}(r)\right)
$$

In the equation, $\overline{r_{E R}(d)}$ represents the mean grayscale intensity value, and $P_{S_{T}(d)}(r)$ is the probability of the pixel whose grayscale intensity value is $r$. The weighted entropy difference between the candidate superpixel patch and the extended neighborhood is:

$$
V\left(S_{T}\right)=\arg \min \left(E\left(S_{T}(d)\right)\right)-E\left(S_{T}\right)
$$

Then, the growth rate of weighted entropy can be represented by $\frac{V\left(S_{T}\right)}{E\left(S_{T}\right)}$. Compare it with the second threshold $T_{2}$ to determine whether the candidate superpixel patch belongs to the target area or the background area, so that the false alarm superpixel patches can be removed, as illustrated in the following expression:

$$
\left\{\begin{array}{l}
\frac{V\left(S_{T}\right)}{E\left(S_{T}\right)}>T_{2}, S_{\mathrm{T}} \text { belongs to the target area } \\
\frac{V\left(S_{T}\right)}{E\left(S_{T}\right)}<T_{2}, S_{\mathrm{T}} \text { belongs to the background }
\end{array}\right.
$$

\subsubsection{The false alarm filtering method based on Harris corner detection}

Harris corner detection algorithm is often used in optical image target detection; however, it is seldom used for target detection in SAR images [14]. The high-resolution marine ship targets often have some corners, and they can be detected. The Harris corner algorithm is used to detect the corner of ship targets in high-resolution SAR images. Then, the corner number threshold $T_{3}$ is set to further filter out the false alarm patches, and the final target detection result in SAR

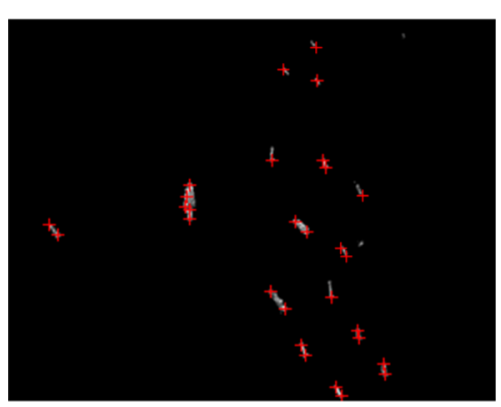

a

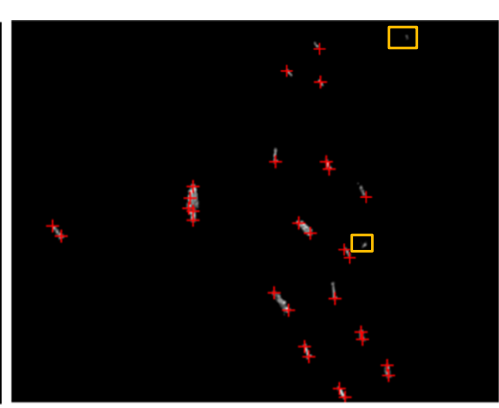

b

Fig. 7 The Harris detection result and false alarm target marking results. a Harris corner detection result. b The false alarm targets marking result 


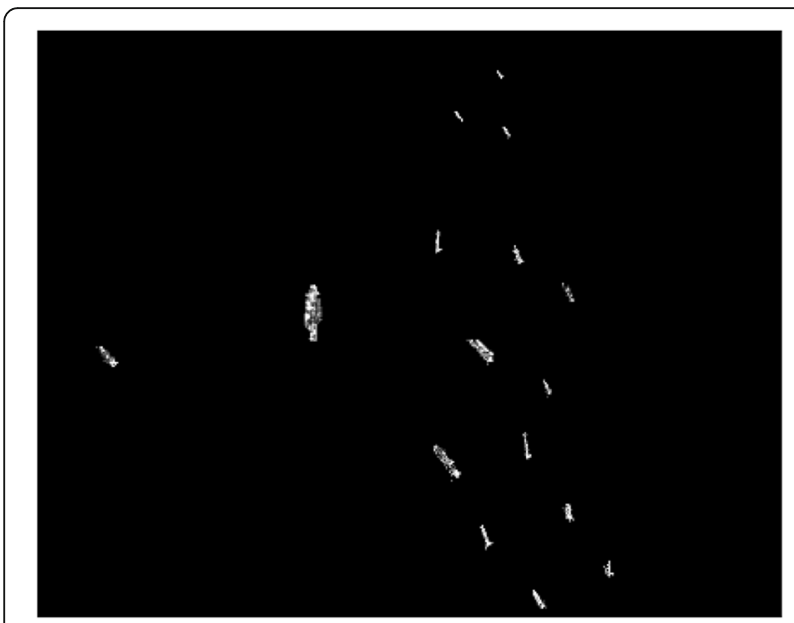

Fig. 8 The final detection result

images can be obtained. If the corner number is more than $T_{3}$, the ship target can be retained; otherwise, it will be filtered.

\section{Experimental design and results}

\subsection{Experiment on ship SAR image with land}

A high-resolution marine ship SAR image with land is selected, as shown in Fig. 3. The resolution is $1.25 \mathrm{~m} \times$ $1.25 \mathrm{~m}$, and its size is $961 \times 762$ pixels; moreover, the SAR image contains 17 ships.

Firstly, Otsu's method combining with the morphological processing method is used to shield the land area in the ship SAR image [15], and the land shielding result is shown in Fig. 4a. Then, the improved SLIC superpixel segmentation algorithm is used to process it. The step size of the patch area is set to 13 pixels, and the superpixel generation result is shown in Fig. $4 \mathrm{~b}$.

The self-information of the superpixel patches is calculated, as shown in Fig. 5 below, and then set the outlier parameter $T_{1}$ as 1.4, and the candidate superpixel patches are shown in Fig. 6a. There are some false alarm superpixel patches in the graph. Then, set the growth rate of the neighborhood weighted information entropy $T_{2}$ as $20 \%$, and the detection result is shown in Fig. $6 \mathrm{~b}$.

Carry out the Harris corner detection on the filtering result of Fig. 6b, and the detection result is shown in Fig. 7a. Then, set the threshold $T_{3}$ as 1 , after filtering out the two false alarm targets marked by yellow rectangle box as shown in Fig. 7b, the final detection result can be obtained as shown in Fig. 8 .

Compare the detection performance of the proposed algorithm with other two detection algorithms, including the VWIE detection algorithm and visual attention with the VA-SVD detection algorithm, and all experiments are conducted in the same Matlab R2016b environment and in the same desktop computer with a processor of Intel Core $i 7-8700 \mathrm{~K}$ and a memory of $32.0 \mathrm{G}$. The detection results based on the two detection algorithms for comparison are shown in Fig. 9.

In Fig. 9, the false alarm targets are marked by the yellow rectangle box and the missing targets are marked by the blue triangles in their corresponding position of the original ship targets. The targets whose form is seriously distorted are marked by the red circles. The figure of merit (FoM) is defined as a quality factor of the detection performance, and $\mathrm{FoM}=\frac{N_{\mathrm{tt}}}{N_{\mathrm{gt}}+N_{\mathrm{fa}}}$. In the equation, $N_{\mathrm{tt}}, N_{\mathrm{fa}}$, and $N_{\mathrm{gt}}$ represent the number of correctly detected targets, the number of false alarm targets, and the number of real targets, respectively. The newly defined detection distortion rate is the ratio of the distorted target number to the total number of ship targets, and $N_{\mathrm{dd}}$ represents the number of the distorted targets. The performance of the three detection algorithms is shown in Table 1.

It can be seen that the detection result of the proposed algorithm has a higher detection rate and a lower missing detection rate, and the false alarm rate and detection distortion rate are both lower. There are some false alarm targets in the detection result of the VA-SVD algorithm even though the ship targets

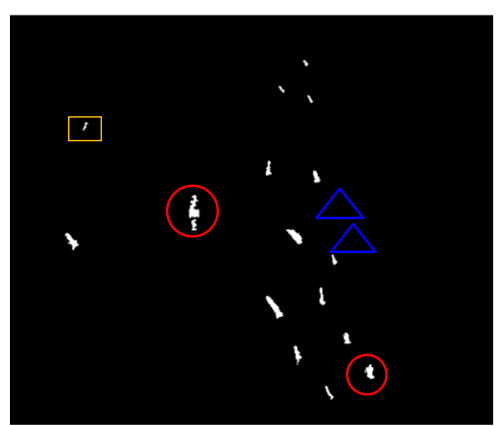

a

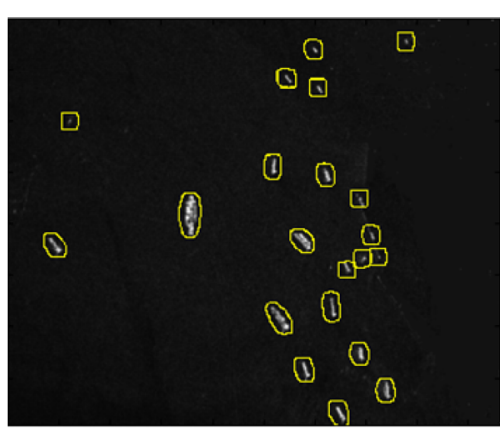

b

Fig. 9 Detection results for comparison. a Detection result of the WWIE algorithm. b Detection result of the VA-SVD algorithm 
Table 1 Comparison of detection performance among the three detection algorithms

\begin{tabular}{|c|c|c|c|c|c|c|c|}
\hline Detection algorithm & $N_{\mathrm{tt}}$ & $\mathrm{N}_{\mathrm{fa}}$ & Number of missing targets & $N_{\mathrm{dd}}$ & Detection distortion rate (\%) & FOM & Time (s) \\
\hline WWIE & 15 & 1 & 2 & 2 & 11.76 & 0.833 & 31.6531 \\
\hline VA-SVD & 17 & 4 & 0 & 0 & 0 & 0.810 & 56.3753 \\
\hline The proposed algorithm & 16 & 0 & 1 & 0 & 0 & 0.941 & 29.0221 \\
\hline
\end{tabular}

are all correctly detected. In addition, the proposed detection algorithm has a higher operation efficiency, and the original shape of the ship targets can be completely retained. Moreover, the ship targets can be generally located and can be shown clearly in the SAR image.

\subsection{Experiment on ship SAR image without land}

When there is no land area in the ship SAR image, there is no need to shield the land area. A high-resolution marine ship SAR image without land is selected, as shown in Fig. 10a. The resolution is $1.25 \mathrm{~m} \times 1.25 \mathrm{~m}$, and its size is $376 \times 319$. It can be seen that the SAR image contains five ships. Since there is no land area in the SAR image, it is not necessary to shield the land area in the ship SAR image. Then, the improved SLIC superpixel segmentation algorithm is used to process the SAR image. The step size of the patch area is set to 13 pixels, and the superpixel generation result is shown in Fig. 10b. Then, calculate the self-information of the superpixel patches, and set the outlier parameter $T_{1}$ as 1.4 ; the candidate superpixel patches are shown in Fig. 10c. It can be seen that there are some false alarm superpixel patches, and then set the growth rate of the neighborhood weighted information entropy $T_{2}$ as 20\%; the detection result is shown in Fig. 10d. Carry out the Harris corner detection on the filtering result of Fig. 10d, the detection result is shown in Fig. 10e, then set the threshold $T_{3}$ as 3 , the final detection result can be obtained as shown in Fig. $10 \mathrm{f}$.

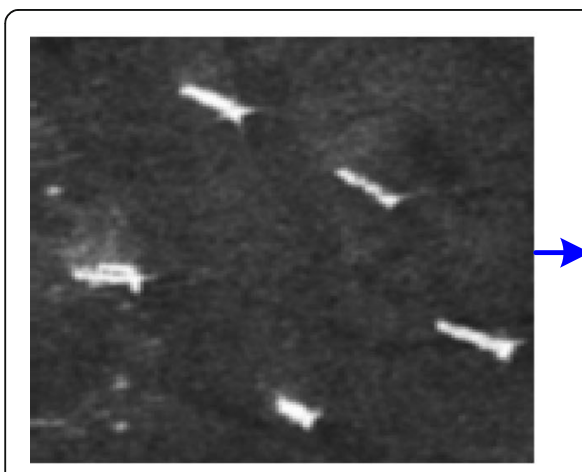

a

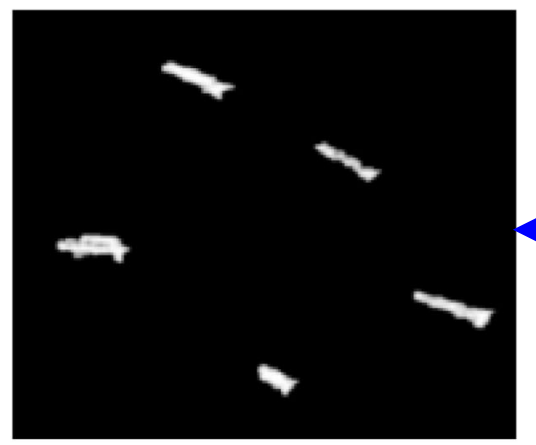

f

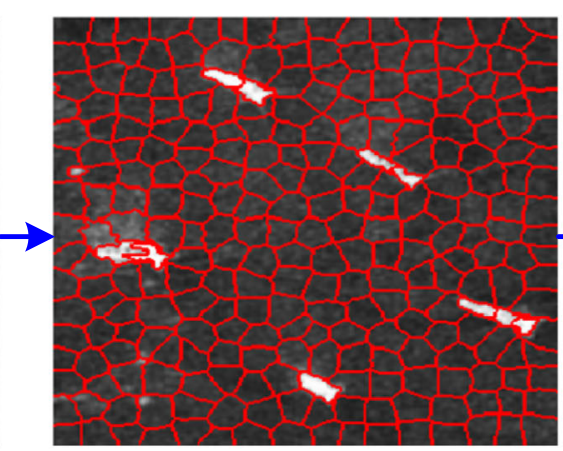

b

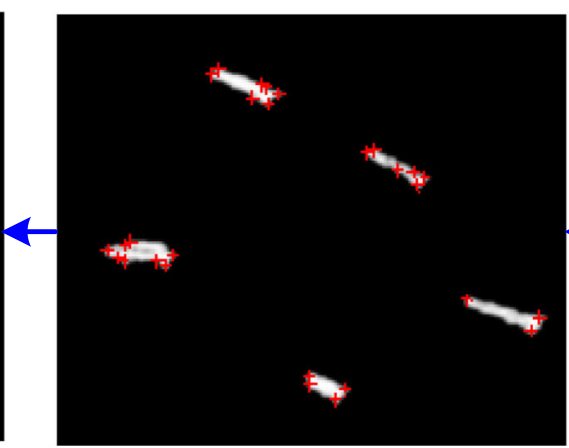

e
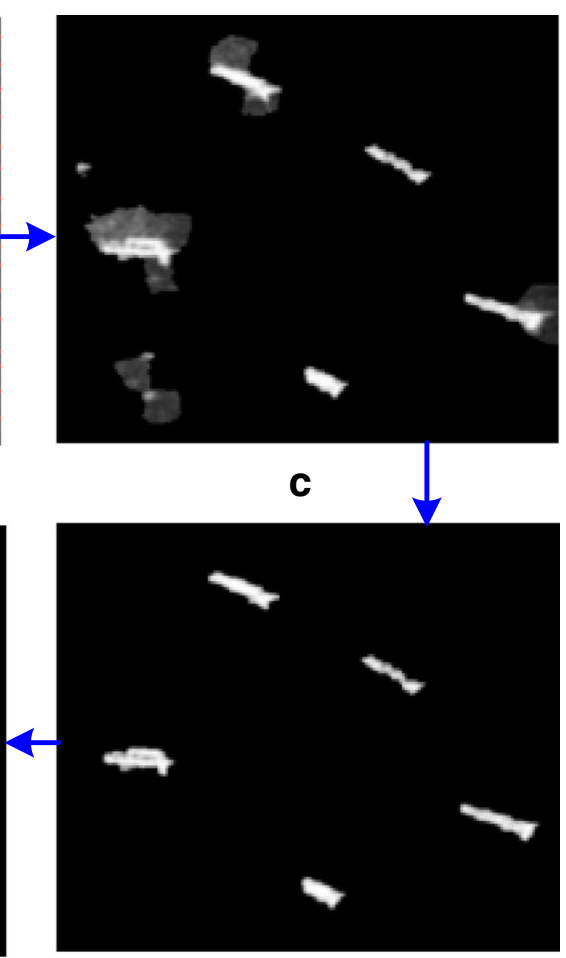

d

Fig. 10 The detection result of the SAR image without land area. a The original SAR image. b Superpixel segmentation result. c The candidate superpixel patches. $\mathbf{d}$ The weighted entropy filtering result. e Harris corner detection result. $\mathbf{f}$ The final detection result 


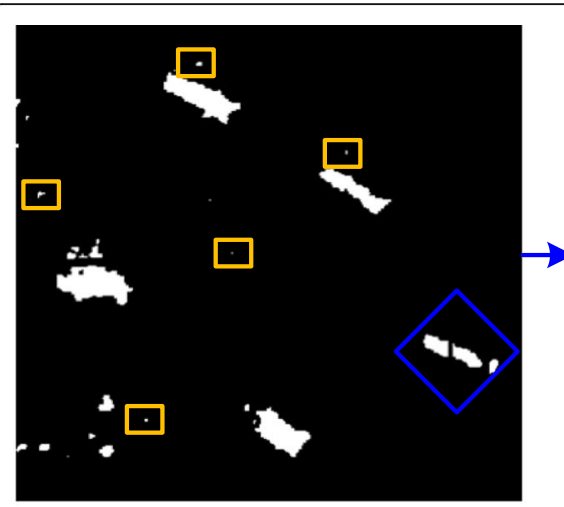

a

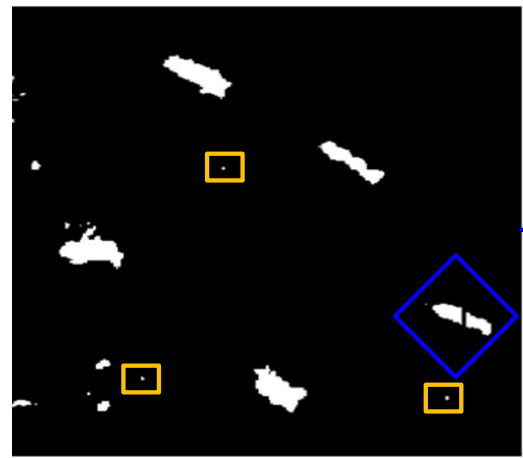

C

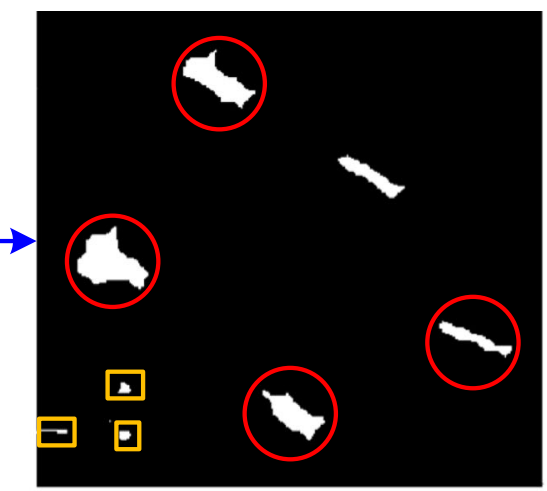

b

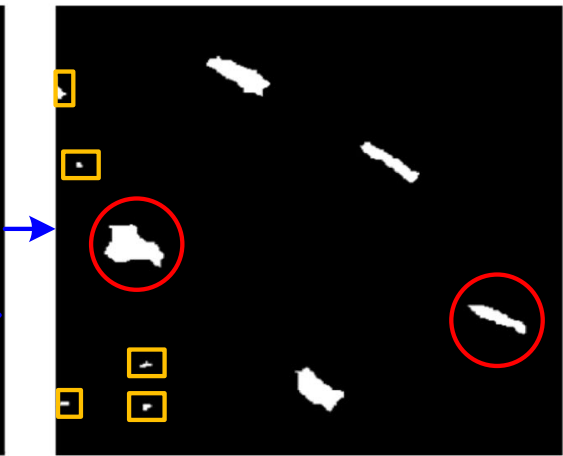

d

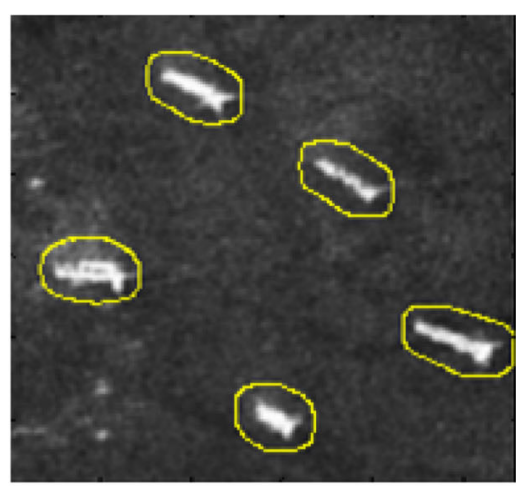

e

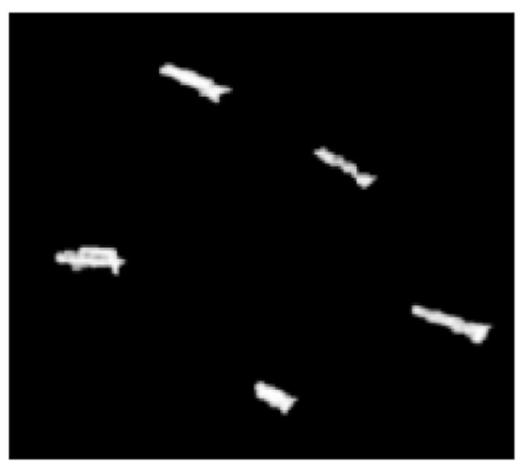

f

Fig. 11 The detection results based on the three detection algorithms for comparison. a CFAR detection result based on gamma distribution. $\mathbf{b}$ Morphological processing. c Detection result based on two-parameter CFAR. d Morphological processing. e VA-SVD detection result. $\mathbf{f}$ The proposed detection result algorithm

Because the false alarm targets have already been filtered out in the former global detection and local detection steps, the Harris corner detection can only be used to mark the location of the ship targets. Compare the detection performance of the proposed algorithm with other three detection algorithms, and all experiments are conducted in the same Matlab R2016b environment. The detection results based on the three detection algorithms for comparison are shown in Fig. 11.

In Fig. 11, the false alarm targets are marked by yellow rectangle boxes, and the broken target is marked by blue rhombus. The targets whose form is seriously distorted are marked by red circles. The performance of the four detection algorithms is shown in Table 2.

In Fig. 11 and Table 2, we can see that even though some false alarm targets can be filtered by using the morphological processing, the original shape of the ship targets cannot be well retained.

\section{Summary and conclusion}

A ship target detection algorithm based on information theory and Harris corner detection for SAR images is proposed in this paper. A self-information-based global detection and a weighed entropy-based local detection can almost separate the ship targets from the background; moreover, the Harris corner detection algorithm is used to further filter out the false alarm targets. The simulation experiments verify the effectiveness and superiority of the proposed algorithm. Not only can a higher detection rate of SAR ship targets and a lower missing detection rate can be ensured, but also it can ensure a lower false alarm rate and a lower detection

Table 2 Comparison of detection performance among the four detection algorithms

\begin{tabular}{|c|c|c|c|c|c|c|c|}
\hline Detection algorithm & $N_{\mathrm{tt}}$ & $N_{\mathrm{fa}}$ & Number of missing targets & $N_{\mathrm{dd}}$ & Detection distortion rate (\%) & FOM & Time (s) \\
\hline Gamma CFAR & 5 & 6 & 0 & 4 & 80 & 0.455 & 6.7276 \\
\hline Two parameter CFAR & 5 & 5 & 0 & 2 & 40 & 0.500 & 7.5361 \\
\hline VA-SVD & 5 & 0 & 0 & 0 & 0 & 1.000 & 3.1749 \\
\hline The proposed algorithm & 5 & 0 & 0 & 0 & 0 & 1.000 & 2.2252 \\
\hline
\end{tabular}


distortion rate. In addition, the proposed detection algorithm has a higher operation efficiency, and the original shape of the ship targets can be completely retained. In subsequent further research, we will combine other features of the ship targets to improve the algorithm for ship target detection in SAR images with a larger scene and richer information. Moreover, adaptive superpixel segmentation will be studied so that the algorithm can segment the SAR image adaptively according to the number and size of the ship targets in the SAR image.

\section{Abbreviations}

CFAR: Constant false alarm rate; FoM: Figure of merit; MWVIE: Multiscale variance weighted image entropy; SAR: Synthetic aperture radar; SLIC: Simple linear iterative clustering; VA-SVD: Visual attention with singular value decomposition; WWIE: Variance weighted information entropy

\section{Funding}

This research is funded by Department of Science and Technology of Sichuan Province (Award Number 2016JY0106) and Education Department of Sichuan Province (Award Number 16ZA0209).

\section{Availability of data and materials}

Data sharing not applicable to this article as no datasets were generated or analyzed during the current study.

\section{Authors' contributions}

YD proposed the study, conducted the experiments, and wrote the manuscript. HW analyzed the data. SL revised the manuscript. MS advised on the study design. $\mathrm{XL}$ advised on the manuscript structure. All authors read and approved the final manuscript.

\section{Competing interests}

The authors declare that they have no competing interests.

\section{Publisher's Note}

Springer Nature remains neutral with regard to jurisdictional claims in published maps and institutional affiliations.

\section{Author details}

${ }^{1}$ College of Electronic Engineering, Chengdu University of Information Technology, Chengdu 610225, Sichuan, China. ${ }^{2}$ Physics Science and Information Engineering College, Liaocheng University, Liaocheng 252000, Shandong, China.

Received: 23 September 2018 Accepted: 11 December 2018 Published online: 20 December 2018

\section{References}

1. M. Yeremy, J.W.M. Campbell, K. Mattar, et al., Ocean surveillance with polarimetric SAR. Can. J. Remote. Sens. 27(4), 328-344 (2001).

2. W. An, C. Xie, X. Yuan, An improved iterative censoring scheme for CFAR ship detection with SAR imagery. IEEE Trans. Geosci. Remote Sens 52(8), 4585-4595 (2014).

3. P.P. Gandhi, S.A. Kassam, Analysis of CFAR processors in homogeneous background. IEEE Trans. Aerosp. Electron. Syst. 24(4), 427-445 (2002).

4. V. Anastassopoulos, G. Lampropoulos, Optimal CFAR detection in Weibull clutter. IEEE Trans. Aerosp. Electron. Syst 31(1), 52-64 (1995).

5. X. Qin, S. Zhou, H. Zou, et al., A CFAR detection algorithm for generalized gamma distributed background in high-resolution SAR images. IEEE Geosci. Remote Sens. Lett 10(4), 806-810 (2013).

6. X. Ren, J. Malik, Learning a classification model for segmentation//IEEE International Conference on Computer Vision, 2003. Proceedings. IEEE, vol 1 (2003), pp. 10-17.

7. W. Yu, Y. Wang, H. Liu, et al., Superpixel-based CFAR target detection for high-resolution SAR images. IEEE Geosci. Remote Sens. Lett. 13(5), 730-734 (2016).
8. B. Hou, W. Yang, S. Wang, et al., SAR image ship detection based on visual attention model//Geoscience and Remote Sensing Symposium. IEEE (2014), pp. 2003-2006.

9. S. Liu, Z. Cao, SAR image target detection in complex environments based on improved visual attention algorithm. Eurasip J. Wireless Commun. Netw. 2014(1), 54 (2014).

10. Z. Wang, L. Du, P. Zhang, et al., Visual attention-based target detection and discrimination for high-resolution SAR images in complex scenes. IEEE Trans. Geosci. Remote Sens. 99, 1-18 (2017).

11. Z. Cao, Y. Ge, J. Feng, Fast target detection method for high-resolution SAR images based on variance weighted information entropy. Eurasip J. Adv. Signal Process 2014(1), 45 (2014)

12. X. Wang, C. Chen, Ship detection for complex background SAR images based on a multiscale variance weighted image entropy method. IEEE Geosc. Remote Sens Lett. 14(2), 184-187 (2017).

13. R. Achanta, A. Shaji, K. Smith, et al., SLIC superpixels compared to state-ofthe-art superpixel methods. IEEE Trans. Pattern Anal. Mach. Intell. 34(11), 2274 (2012).

14. C. Harris, A combined corner and edge detector. Proc Alvey Vision Conf 1988(3), 147-151 (1988).

15. Q. Wang, Inshore ship detection using high-resolution synthetic aperture radar images based on maximally stable extremal region. J. Appl. Remote. Sens. 9(1), 095094 (2015).

\section{Submit your manuscript to a SpringerOpen ${ }^{\circ}$ journal and benefit from:}

- Convenient online submission

- Rigorous peer review

- Open access: articles freely available online

- High visibility within the field

- Retaining the copyright to your article

Submit your next manuscript at $\boldsymbol{\nabla}$ springeropen.com 